American Journal of Pharmaceutical Education 2017; 81 (5) Article 88.

\title{
RESEARCH
}

\section{Assessing Faculty and Student Interpretations of AACP Survey Items with Cognitive Interviewing}

\author{
Samuel C. Karpen, PhD, Nicholas E. Hagemeier, PharmD, PhD \\ Gatton College of Pharmacy, East Tennessee State University, Johnson City, Tennessee \\ Submitted March 23, 2016; accepted September 16, 2016; published June 2017.
}

Objective. To use cognitive interviewing techniques to determine faculty and student interpretation of a subset of items from the AACP faculty and graduating student surveys.

Methods. Students and faculty were interviewed individually in a private room. The interviewer asked each respondent for his/her interpretation of 15 randomly selected items from the graduating student survey or 20 items from the faculty survey.

Results. While many items were interpreted consistently by respondents, the researchers identified several items that were either difficult to interpret or produced differing interpretations.

Conclusion. Several interpretational inconsistencies and ambiguities were discovered that could compromise the usefulness of certain survey items.

Keywords: AACP faculty survey, AACP graduating student survey, cognitive interview, qualitative methods, measurement error

\section{INTRODUCTION}

Surveys are a common data collection instrument in the health sciences. Indeed, nearly $80 \%$ of the original research reports published in the Journal of Graduate Medical Education in 2011 and 2012 included surveys in the design, ${ }^{1}$ and a recent search for "survey" on the American Journal of Pharmaceutical Education (AJPE) website yielded 1,433 hits. Given the prevalence of surveys in health professions education, students and practitioners should understand and apply standard practices in survey design. This article introduces cognitive interviewing as a step in the survey design process for improving a survey's validity.

Cognitive interviewing is a technique for eliciting respondents' thoughts as they answer survey items, and to gain insight into their interpretations, answering strategies, and difficulties. ${ }^{2,3}$ Given its ability to identify interpretational difficulties and inconsistencies, several federal agencies, including the US Census Bureau, National Center of Health Statistics, and Bureau of Labor Statistics, regularly employ cognitive interviewing, and some agencies even have dedicated cognitive interviewing labs. Furthermore, recent articles have encouraged graduate medical educators to employ more rigorous survey pre-testing and design processes, including cognitive

Corresponding Author: Samuel C. Karpen, Building 7, Maple Avenue, James H. Quillen VA Medical Center Campus, Johnson City, TN 37614-1704. Tel: 423-439-6883.

E-mail: karpen@mail.etsu.edu interviewing. ${ }^{1,4,5}$ For example, Magee and colleagues, and Richards and colleagues list a series of questions that graduate medical educators should ask themselves when designing a survey. One question: "Will my respondents interpret my items in the manner that I intended" is directly related to cognitive interviewing. In fact, both authors encourage cognitive interviewing and describe its importance as follows: "This process is invaluable in identifying problems with question or response wording that may result in misinterpretations or bias. Ultimately, the goal is twofold: to ensure respondents understand the questions as you intended and to verify that different respondents interpret the items the same way and can respond to the items accurately."

Nursing educators have also encouraged cognitive interviewing as a means of improving survey validity. ${ }^{6-8}$ Izumi and colleagues used it to develop the Quality of Nursing Care Index (QNCI), an instrument that measures the quality of nursing care from the perspective of patients with advanced stage illnesses, and uncovered several confusing and potentially offensive items as a result. ${ }^{8}$ Likewise, Ahmed and colleagues submitted a palliative care screening measure to cognitive interviewing and found that several items were either confusing or contained jargon. ${ }^{6}$

While cognitive interviewing is rare in pharmacy education, the field does employ several surveys that may be good candidates for cognitive interviewing. Specifically, in collaboration with an (ACPE) task force, the American Association of Colleges of Pharmacy (AACP) 


\section{American Journal of Pharmaceutical Education 2017; 81 (5) Article 88.}

developed multiple stakeholder surveys - graduating student, faculty, preceptor, and alumni surveys - that are made available to schools/colleges on an annual basis. AACP's Office of Institutional Research and Effectiveness indicates the purpose of the surveys is to "help member colleges and schools gather data for both continuous program improvement and accountability (accreditation) purposes." Whereas individual colleges and schools have discretion in the timing and frequency of the survey administration, the results thereof are part of the college/ school ACPE self-study and the accreditation process. In addition to their utility in the accreditation process, survey results inform program-level internal decision making.

These surveys however, have never undergone cognitive interviewing. ${ }^{9}$ While the AACP faculty and student surveys were validated, the means of validation were quantitative, which do not always detect interpretational differences. For example, an item could have been interpreted consistently by most respondents but differently than the researchers had intended. Alternatively, an item could have been answered similarly by most respondents despite differing interpretations. In either case, the item would have displayed acceptable psychometric properties. We submitted portions of both instruments to cognitive interviewing to determine whether interpretational differences existed that may not have been detected by purely quantitative means. In doing so, we also introduced cognitive interviewing to pharmacy educators.

The typical sample size for a cognitive interview is five to ten participants, ${ }^{2,3}$ thus, we recruited five students and five faculty to participate in the interviews from a southeastern college of pharmacy. Participants were recruited based on their response to a general invitation made to all faculty and enrolled students; they were not randomly selected, as less of a priority is placed on generalization in small sample qualitative research. ${ }^{2,3,10,11}$

That said, we included faculty at all ranks from both the pharmaceutical sciences and pharmacy practice departments, and students intending to pursue a variety of specialties, (eg, community pharmacy, ambulatory care), career paths (eg, direct practice entry, residency training), and degrees (eg, PharmD and PharmD/Master of Public Health). Approval was obtained from the IRB prior to the interviews.

\section{METHODS}

Students and faculty were interviewed individually in a private room. After obtaining consent, the interviewer asked the participant to spend five minutes describing the first floor of the participant's house in order to acclimate him/her to verbalizing thoughts to the interviewer. Additionally, the interviewer asked questions such as, "How many windows are in your first floor" or "Do you consider glass areas of doors to be windows" to acclimate the participant to verbal probing. Due to time constraints, the interviewer did not use AACP survey in its entirety; rather, two items were randomly selected from each section of each survey with the aid of a random number generator, resulting in 15 items from the graduating student survey and 20 items from the faculty survey. After the warm-up exercise, the interviewer handed the participant a printed copy of the pre-selected survey items and asked the first question. The interviewer primarily relied on the think aloud method, but occasionally asked participants to define terms or to rephrase the question via probes. The interviewer took notes while participants were speaking. All faculty and student interviews required about 45 minutes to complete.

As the name suggests, think aloud interviewing requires participants to relay their thoughts to the interviewer while answering each survey item. The interviewer simply records the participant's thoughts and interjects little more than "Tell me more" or "Tell me what you're thinking." During verbal probing, the interviewer asks questions targeted at specific aspects of a survey item. For example, an interviewer may ask a participant how he/she arrived at a particular answer, why he/she hesitated before providing an answer, or what a particular term means to him/ her. In-depth reviews of each of these techniques, as well as discussions of their advantages and disadvantages, are widely available. . $^{2,5,12,13}$

After the interviews, participants' difficulties - if any - were coded into one of five categories: lexical, inclusion/exclusion, temporal, logical, and computational. ${ }^{14}$ Lexical difficulties concern understanding the intended meaning or usage of words or phrases within an item. Inclusion/exclusion also deals with word meaning, but specifically focuses on which concepts are included within the scope of a word or phrase. For example, a participant may consider dentists when asked about doctors, even though the researcher had intended to ask only about MDs. Temporal difficulties concern interpretations of the range or amount of time spent on the activity described in the question. Logical problems cover a wide range of difficulties, from improper use of conjunctions, to multiple barreled questions, to leading questions. Computational problems typically function as an "other" category when a problem does not fit into the first four categories.

Participants' difficulties were also coded into one of three response stages: understanding, task performance, and response formatting. ${ }^{6}$ Understanding concerns participants' ability to comprehend the meaning of the question, while task 


\section{American Journal of Pharmaceutical Education 2017; 81 (5) Article 88.}

Table 1. Faculty Responses to Survey Items

Question
$\begin{aligned} & \text { The college/school's administrator(s) are } \\ & \text { responsive to my needs/problems. }\end{aligned}$
responsive to my needs/problems.

The performance feedback I receive is effective.

I receive adequate support staff resources.

Computer resources are adequate for my academic responsibilities.

The college/school effectively employs strategic planning.

The PharmD prepares students to effectively manage a patient-centered pharmacy practice.

\section{Summary of Responses}

Respondents were unsure who to consider administrators. Some only considered the dean and associate deans, while others included directors. Some included the department chairs, and others did not. Respondents also had differing interpretations of responsiveness. Some thought that in order to be responsive, the administrators were required to meet/solve their needs and problems, while others considered mere acknowledgement a form of responsiveness. Other participants were unsure how to define responsiveness.

Participants had varying interpretations of effective and feedback. Some defined effective as "actionable" or "something I can use" while others defined effective as "motivates me to do better" or "boosts my morale and helps to retain me," and others were unsure how to define "effective." Participants' interpretation of "feedback" was similarly disparate, as some only considered formal meetings with their chair, while others considered casual conversations. Some considered student evaluations, and others did not.

Participants disagreed on who qualified as "support staff" and what was meant by adequate. Most participants only considered office managers and secretaries support staff, but others included the technology manager, and the directors of academic affairs and assessment. Some participants thought that adequate referred to whether or not the college had an adequate number of support staff, while others thought that adequate referred to whether or not the existing support staff were appropriately competent.

Respondents did not agree on the definition of "computer resources"; some only considered software, others only considered hardware, and others considered access to library resources and online publications.

Participants were unsure of the definitions of "strategic planning" and "effective." One was unsure whether "strategic planning" referred to hiring, fundraising, or retention. Another was unsure whether "effective" meant that the plan was actionable or that we simply had a plan.

Several faculty rephrased this question as, "Can our students manage a pharmacy practice." Others were unsure whether "manage" referred to patient care, business operations, or both. One said that she would answer the question differently depending on her definition of manage, because "the college teaches students to manage patient care, but not personnel." 


\section{American Journal of Pharmaceutical Education 2017; 81 (5) Article 88.}

Table 1. (Continued)

Question
The PharmD prepares students to
demonstrate expertise in the area of
informatics (resources, devices, and
methods required to optimize the
acquisition, storage, retrieval,
and use of information in pharmacy
and health care).

I am given the opportunity to provide evaluative feedback

of the administrators.

My allocation of effort has been clearly stated.

Criteria for my performance assessment are consistent with my responsibilities.

The college/school has a sufficient number of qualified faculty.

The physical facilities enable out-of-class interaction among administrators, faculty, and students.

Programs are available to me to improve my teaching and to facilitate student learning.

Programs are available to me that help me develop my competence in research and/or scholarship.
Summary of Responses

Participants had different interpretations of this question. One rephrased it as "Do we prepare students to navigate electronic medical records and large databases on drug and public health information?" while another rephrased it as "Do we prepare students to use drug information resources?" Two faculty members were unsure why they should be teaching students to use the latest non-pharmacy related hardware. One asked, "Shouldn't the question be asking about relevant things like insulin pumps?" One thought that "informatics" was a buzzword with no clear meaning.

While most respondents thought that this item sounded like a yes/no question, there was little interpretive ambiguity.

Since our faculty state their own allocation in a document that is later reviewed by the chairs, many struggled with this item. For example, two stated: "Faculty state allocation that is later reviewed by chair, so question is hard to interpret" and "This is unclear because chairs don't assign allocation up front. Faculty report allocation and chairs advise."

Respondents' interpretation of this item varied somewhat. Some read it as, "Am I aware of the scale that I'm being evaluated on" while others read it as "Am I able to meet the requirements for tenure" and others read it as "Am I evaluated on what I spend time doing." Also, faculty were unsure what "performance assessment" meant. One said, "Does it mean, my annual review, my course evaluations, peer evaluations, or all of the above?"

Respondents all thought that this item was clear.

While everyone understood the question, several respondents commented that faculty and administrators only interact outside of class.

Many respondents were unsure whether the question was about the availability of one program that both improved teaching and facilitated student learning or separate programs: one that improved teaching and one that facilitated student learning.

Respondents all thought that this item was clear. 


\section{American Journal of Pharmaceutical Education 2017; 81 (5) Article 88.}

Table 1. (Continued)

\begin{tabular}{|c|c|}
\hline Question & Summary of Responses \\
\hline $\begin{array}{l}\text { Laboratories and other non-classroom } \\
\text { environments are conducive } \\
\text { to learning. }\end{array}$ & $\begin{array}{l}\text { Some faculty were unsure how to define "conducive to learning." "Does } \\
\text { it mean that learning could occur there, or that the room is set up } \\
\text { for learning?" Some interpreted conducive as "quiet" while others } \\
\text { discussed lab equipment. Additionally, respondents were unsure } \\
\text { what qualified as a non-classroom environment. Examples included } \\
\text { labs, meeting rooms, bathrooms, lounges, and study rooms. } \\
\text { Additionally, "conducive to learning" was perceived to } \\
\text { be room specific. }\end{array}$ \\
\hline $\begin{array}{l}\text { The curriculum is consistent with the } \\
\text { collective vision of the faculty } \\
\text { and administration. }\end{array}$ & $\begin{array}{l}\text { Some faculty thought that this item was difficult to answer because } \\
\text { while everyone approves the curriculum, not everyone envisions } \\
\text { it. One common way to interpret the question was, "Is the } \\
\text { curriculum consistent with our Vision Statement?" }\end{array}$ \\
\hline $\begin{array}{l}\text { The PharmD program prepares } \\
\text { students to communicate with patients, } \\
\text { caregivers, and other members } \\
\text { of the inter-professional health }\end{array}$ & $\begin{array}{l}\text { While several faculty commented that students were not trained } \\
\text { equally well to communicate with each of the three groups } \\
\text { mentioned in the question, there were no interpretational difficulties. }\end{array}$ \\
\hline
\end{tabular}

care team.

The PharmD program prepares students to maintain professional competence.

The college/school provides an environment and culture that promotes professional behavior among students, faculty, administrators, preceptors, and staff.

Respondents all thought that this item was clear.

In my opinion, the proportion of my time spent on teaching is:

Some respondents struggled to answer this question because they did not believe that professionalism was promoted equally among each of the five groups mentioned in the question. Furthermore, some respondents reported the college's culture and environment did not promote professionalism equally well. performance concerns their ability to generate an internal response, and response formatting concerns their ability to map the internal response to an available response option.

Tables based on this coding scheme sometimes accompany cognitive interviews as a brief quantitative summary of the number and type of problems detected. This information is not typically used to diagnose problems with specific items or suggest solutions; for that, researchers should look to the participants' comments. Some researchers, however, find this coding scheme helpful, as it provides a way to understand the problems inherent in a survey with a brief glance, and also allows them to quantitatively compare revisions of a survey.

\section{RESULTS}

Results of the faculty interviews are presented first (Table 1), followed by results of the student interviews
(Table 2). The format for both is a restatement of the original question, followed by a summary of participants' comments. The coded responses are presented at the end of the section.

Faculty Summary: Several questions elicited conflicting and uncertain interpretations from faculty, often as a result of multiple barreled items, and vague terms, like "administrators," "student affairs," and "continuity of care." These issues are reflected in Table 3 where most problems fell into Lexical and Omission/Inclusion at the Understanding stage, and Logic at the Task Performance state. For example, faculty were unsure who counted as administrators (Q1) or support staff (Q3), hence these types of difficulties were coded as UnderstandingOmission/Inclusion. Another common issue was multiple barreled questions. For example, Question 17 required respondents to evaluate three different groups. Many respondents were unsure which group they should 


\section{American Journal of Pharmaceutical Education 2017; 81 (5) Article 88.}

Table 2. Student Responses to Survey Items

\begin{tabular}{l} 
Question \\
\hline I developed the skills needed to \\
prepare me for continued learning \\
after graduation. \\
The college/school of pharmacy made \\
use of a variety of means (eg, course \\
evaluations, student surveys, focus \\
groups, meetings with administrative \\
leaders) to obtain student perspectives \\
on curriculum, student services, faculty \\
student relationships and other aspects \\
of the program.
\end{tabular}

In the ambulatory care setting, I was able to apply my patient care skills.

The PharmD program prepared me to work with other stakeholders (eg, patients and other health professionals) to engender a team approach to ensure appropriate use of health care resources in providing patient care.

The learning experience with other professions students helped me to gain a better understanding of how to be part of a multi-disciplinary team to improve patient outcomes.

The sites available for introductory pharmacy practice experiences were high quality.

The need for continuity of care throughout the health care system was emphasized in the advanced pharmacy practice experiences.

The college/school's administration responded to problems and issues of concern to the student body.

Overall, preceptors provided me with individualized instruction, guidance and evaluation that met my needs as a doctor of pharmacy student.

\section{Summary of Responses}

All participants believed that they had developed the skills necessary to continue learning prior to pharmacy school, and were thus unsure of how to answer the question.

Each participant defined "student services" differently, and several commented that they would not answer the same way for student services, faculty student relationships, and the curriculum. Interpretations of "student services" ranged from printers, to lounges, to mental health services, to the library, to fun activities hosted by the college. One student was unsure whether student services referred to services performed by the students for the college or services performed by the college for the students.

Respondents interpreted this item consistently; however, one was unsure whether to define "ambulatory care" by location (eg, physician's office or outpatient clinic) or type of activity performed (eg, counseling patients).

Students held different definitions of health care resources. Some believed that it referred to money, while others believed that it referred to services for the under-privileged, and others believed that it referred to medical personnel and devices.

Though there was some disagreement about whether or not to include didactic experiences in the "learning experiences" described above, students generally interpreted this item in the same manner.

Students interpreted this item similarly.

Students interpreted this item similarly.

Some students thought that faculty were included in "administration" and others only counted the dean and associate/assistant deans. Also, students' interpretations of "problems and issues of concern" varied widely from small annoyances like a non-functioning printer to more severe issues concerning financial aid and academic misconduct.

Students interpreted this item similarly. 


\section{American Journal of Pharmaceutical Education 2017; 81 (5) Article 88.}

Table 2. (Continued)

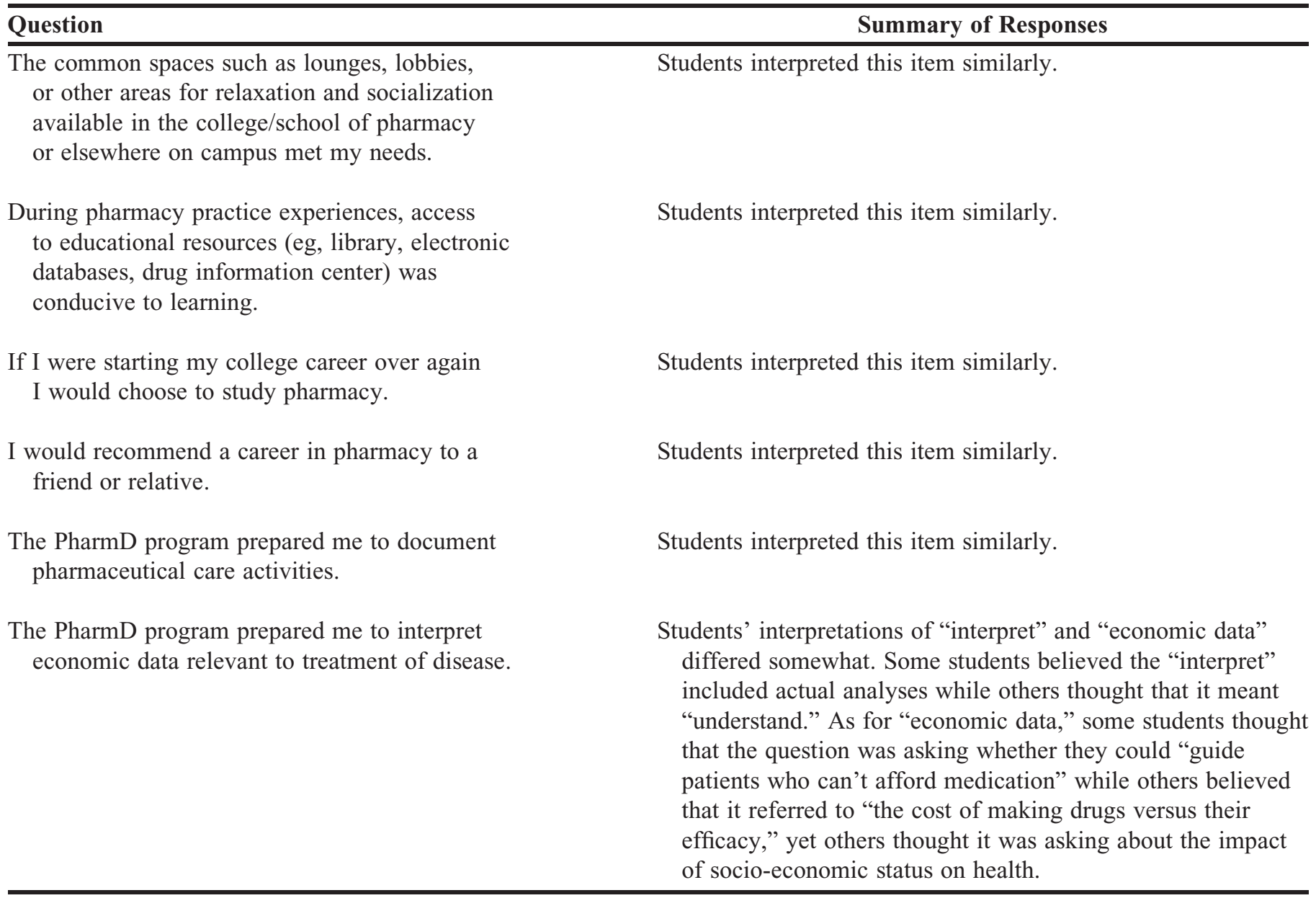

consider most heavily, hence the large proportion of issues recorded under Task Performance-Logical. Finally, the high proportion of problems under Lexical-Understanding indicates that respondents were unsure of the definition of some of the words used in the survey. For example, many faculty were unsure of the intended definition of "conducive to learning" (Q15), "performance assessment” (Q10), and "informatics” (Q7).

Table 3. Number and Type of Problems Revealed During Faculty Cognitive Interviews

\begin{tabular}{lccc}
\hline & \multicolumn{3}{c}{ Response Stage } \\
\cline { 2 - 4 } Problem & \multicolumn{3}{c}{ Task } \\
Type & Understanding & $\begin{array}{c}\text { Response } \\
\text { Performance }\end{array}$ & Formatting \\
\hline Lexical & 17 & 7 & \\
Temporal & & 18 & 2 \\
Logical & & 3 & 3 \\
Computational & 18 & & \\
Omission/ & & & \\
$\quad$ Inclusion & & & \\
\hline
\end{tabular}

Student Summary: Students generally provided less feedback than faculty during the interviews, hence the smaller numbers in Table 4. While the reason for this is unclear, it may be because faculty have had more experience with the survey, and hence more time to think about it. None of the students had taken the Graduating Student Survey, but all faculty had completed the Faculty Survey multiple times. Despite the difference in total number of

Table 4. Number and Type of Problems Revealed During Student Cognitive Interviews

\begin{tabular}{|c|c|c|c|}
\hline \multirow[b]{2}{*}{$\begin{array}{l}\text { Problem } \\
\text { Type }\end{array}$} & \multicolumn{3}{|c|}{ Response Stage } \\
\hline & Understanding & $\begin{array}{c}\text { Task } \\
\text { Performance }\end{array}$ & $\begin{array}{c}\text { Response } \\
\text { Formatting }\end{array}$ \\
\hline $\begin{array}{l}\text { Lexical } \\
\text { Temporal }\end{array}$ & 13 & 1 & \\
\hline Logical & & 7 & 2 \\
\hline Computational & 2 & & \\
\hline $\begin{array}{l}\text { Omission/ } \\
\text { Inclusion }\end{array}$ & 9 & & \\
\hline
\end{tabular}




\section{American Journal of Pharmaceutical Education 2017; 81 (5) Article 88.}

responses between faculty and students, their patterns of difficulties were similar, with most issues falling into Understanding-Lexical, Understanding-Omission/Inclusion, and Task Performance-Logical. Again, most of these issues arose from multiple barreled items, and vague terminology. For example, students were unsure who counted as "administrators" (Q8) or what counted as "student services" (Q3), hence these types of difficulties were coded as Understanding-Omission/Inclusion. While the Student Survey also contained multi-barreled questions (Q2, Q4, Q10), fewer participants commented on them. Finally, the high proportion of problems under LexicalUnderstanding indicates that respondents were unsure of the definition of some of the words used in the survey. For example, many students were unsure of the intended definition of "interpret economic data" (Q13) or "health care resources" (Q14).

\section{DISCUSSION}

This article presents the results of a pilot study evaluation of a formal cognitive interview protocol. Our overarching objectives were to introduce the methodology and to demonstrate its utility using commonly employed survey instruments. Importantly, this article reports results from a limited sample - one college of pharmacy. Our study was not intended to be exhaustive or generalizable to all faculty and students. While none of the items that we tested contained fatal flaws, we noted interpretational inconsistencies and ambiguities that warrant consideration when using the surveys for QI and/or accreditation purposes. For example, if faculty have trouble defining "strategic planning," their responses to items about strategic planning may not be informative. If students do not consistently interpret "student services," administration may not know what to change in the event of declining satisfaction. If faculty do not agree on whether "adequate" refers to the quality or the amount of support staff, overworked support staff may be unjustly reprimanded. A survey's validity will be compromised to the extent that respondents interpret the items inconsistently, struggle to interpret them at all, or interpret them differently than the survey's creators intended.

When cognitive interviews uncover interpretational inconsistencies or difficulties, researchers can look to the participants for guidance. While multiple barreled items, vague terms, and jargon should be avoided whether or not cognitive interviews have been conducted, there are few general solutions to interpretational difficulties or inconsistencies; each survey item is unique. When a participant struggles to interpret a word or phrase, the interviewer can explain the intended meaning and inquire about potential clarifications. If a majority of participants provide the same clarification, it can guide the interviewer's revisions. Similarly, if an interviewer finds inconsistent interpretation of a word or phrase, he or she can ask participants to suggest a rephrasing that elicits the desired interpretation. As cognitive interviewing is typically an iterative process, interviewers can test the suggested revisions during subsequent interviews to determine whether interpretational difficulties and inconsistencies have decreased.

Additionally, there are tools that screen survey items for vague terms and jargon. These tools can be used in conjunction with cognitive interviews, or in place of cognitive interviews if time or resources are limited. For example, Question Understanding Aid (University of Memphis, Memphis, TN $)^{5-17}$ is an online interface that analyzes survey items for jargon, complexity, and clarity. The survey creator types the question or item into a text box, and QUAID returns an assessment of its interpretability. The American Association for Public Opinion Research (AAPOR) also provides helpful question wording resources on its website that researchers can consult if they do not have the time or resources to conduct formal pre-testing. ${ }^{18}$ For readers interested in further training in cognitive interviewing, both AAPOR and the Joint Program in Survey Methodology (JPSM) routinely offer short courses.

\section{CONCLUSION}

Government agencies, and more recently academia, ${ }^{4,5,19}$ have recognized the value of cognitive interviewing in developing valid surveys. Our findings suggest that academic pharmacy may see similar benefits. Future research is warranted to more fully evaluate the manner in which AACP survey items are interpreted and how cognitive interviewing can be employed to help colleges and schools of pharmacy and the Academy effectively and efficiently engage in quality improvement.

Quality improvement need not be limited to surveys, as cognitive interviewing is applicable to any instrument that requires interpretation. ${ }^{2}$ Hence, it could be used for forms, exam questions, or admission interview questions. To date, no research has addressed whether cognitive interviewing improves exams' psychometric properties. Given the emphasis on valid and reliable assessments in the AACP Standards 2016, such research would be both timely and practical. We hope that the information and resources presented herein will encourage pharmacy educators to adopt cognitive interviewing for surveys, and to explore its usefulness for exams, admissions interviews, and any other instruments that require interpretation. 


\section{American Journal of Pharmaceutical Education 2017; 81 (5) Article 88.}

\section{REFERENCES}

1. Rickards G, Margee C, Artino AR. Your can't fix by analysis what you've spoiled by design: developing survey instruments and collecting validity evidence. J Grad Med Educ. 2012;4(4):407-410.

2. Willis GB. Cognitive Interviewing: A Tool for Improving Questionnaire Design. Thousand Oaks, CA: Sage; 2005.

3. Willis GB, Royston P, Bercini D. The use of verbal report methods in the development and testing of survey questionnaires. Appl Cogn Psychol. 1991;5(3):251-267.

4. Magee C, Rickards G, Byars LA, Artino AR. Tracing the steps of survey design: a graduate medical education research example. $J$ Grad Med Educ. 2013;5(1):1-5.

5. Willis GB, Artino AR. What do our respondents think we're asking? Using cognitive interviewing to improve medical education surveys. J Grad Med Educ. 2013;5(3):353-356.

6. Ahmed N, Bestall JC, Payne SA, Noble B, Ahmedzai SH. The use of cognitive interviewing methodology in the design and testing of a screening tool for supportive and palliative care needs. Support Care Cancer. 2009;17(6):665-673.

7. Beck SL, Towsley GL, Berry PH, Brant JM, Smith EM. Measuring the quality of care related to pain management: A multiple-method approach to instrument development. Nurs Res. 2010;59(2):85-92.

8. Izumi S, Vandermause R, Benavides-Vaello S. Adapting cognitive interviewing for nursing research. Res Nurs Health. 2013;36(6):623-633.

9. Plaza CM, Patton JM, Kelley KA, Taylor DA. Principles of Good Use for the AACP Curriculum Quality Perception Surveys. Alexandria, VA: American Association of Colleges of Pharmacy; 2014.

10. Horsburgh D. Evaluation of qualitative research. J Clin Nurs. 2003;12(2):307-312.

11. Kruger RA, Casey MA. Focus Groups: A Practical Guide for Applied Research. $5^{\text {th }}$ ed. Thousand Oaks, CA: Sage; 2014.
12. Beatty PC, Willis GB. Research synthesis: the practice of cognitive interviewing. Public Opin Q. 2007;71(2):287-311. 13. DeMaio TJ, Landreth A. Do different methods produce different results? In: Presser S, Rothgeb JM, Couper MP, Lessler JT, Martin E, Martin J, Singer E, eds. Methods for Testing and Evaluating Survey Questionnaires. Hoboken, NJ: Wiley and Sons; 2004.

14. Bolton RN, Bronkhorst TM. Questionnaire pretesting: computer assisted coding of concurrent protocols. In: Schwarz N, Sudman S, eds. Answering Questions: Methodology for Determining Cognitive and Communicative Processes in Survey Research. San Francisco, CA: Jossey-Bass; 1996.

15. Graesser AC, Cai Z, Louwerse MM, Daniel F. Question understanding aid (QUAID): a web facility that that tests question comprehensibility. Public Opin Q. 2006;70(1):3-22.

16. Graesser AC, Wiemer-Hastings K, Kreuz R, Wiemer-Hastings P, Marques K. QUAID: a questionnaire evaluation aid for survey methodologists. Behav Res Methods Inst Comp. 2000;32(2):254262.

17. Graesser A. QUAID - IIS. University of Memphis Institute for Intelligent Systems website. http:/www.memphis.edu/iis/ projects/quaid.php. Updated January 26, 2016. Accessed May 27, 2016.

18. Question wording. American Association for Public Opinion Research website. https://www.aapor.org/Education-Resources/ForResearchers/Poll-Survey-FAQ/Question-Wording.aspx. Updated September 2007. Accessed May 27, 2016.

19. Haeger H, Lambert AD, Kinzie J, Gieser J. Using cognitive interviews to improve survey instruments. Paper presented at: The Association of Institutional Research 2012 Annual Conference; June 2-6, 2012; New Orleans, LA. http://cpr.indiana.edu/uploads/ AIR2012\%20Cognitive\%20Interviews.pdf. Accessed July 6, 2016. 